\title{
Considerations for the Acquisition of Very Large Area EDS Spectral Image Mosaics
}

\author{
Stephen M Seddio ${ }^{1}$ and Paul K Carpenter ${ }^{2}$
}

1. Thermo Fisher Scientific, Fitchburg, WI USA.

2. Washington University in St. Louis, Dept. of Earth and Planetary Sciences, St. Louis, MO USA.

Modern SEMs, EDS detectors, and microanalysis software have enabled the acquisition of EDS spectral images over very large areas so that entire samples may be described by composition and phase maps (e.g., [1]). Here, we investigate what count richness (X-ray counts/pixel) is needed to meaningfully extract different map types (counts, quantitative, and phase maps) from spectral image mosaic datasets.

The sample of this study is a petrographic thick section (see [1] for more details). Analysis was done in an FE-SEM with an accelerating voltage of $15 \mathrm{kV}$ a probe current of $\sim 12 \mathrm{nA}$. X-rays were detected using dual (a $10 \mathrm{~mm}^{2}$ and a $30 \mathrm{~mm}^{2}$ active area) UltraDry EDS detectors. EDS spectral images were acquired in a $3 \times 4$ grid over the sample and processed into $732 \times 726$ pixel montages of net counts maps, quantitative (wt\%; standardless analysis) elemental maps, and phase maps with a resolution of 2.955 $\mu \mathrm{m} /$ pixel using the Thermo Scientific Pathfinder microanalysis system. In order to generate montages from spectral images with varying counts/pixel, the grid acquisition was repeated 8 times over the same area of the sample. The counts/pixel of each spectral image was varied by adjusting for how long the beam would raster over the mapped area during the acquisition of each spectral image - in so doing, the EDS data at each pixel in each spectral image was acquired with the same time constant (1.6 ms) and dead time $(31 \%)$. Phase maps were extracted from the spectral images using the COMPASS algorithm for multivariate statistical analysis [2,3]. ImageJ (Fiji) v. 1.50a [4] was used to generate difference images showing the pixels that were different between the phase maps of maximum counts/pixel (5228 counts/pixel) and the phase maps of lesser count richness. The "percent difference" (Fig. 2, bottom) was calculated as the number of different pixels for each of the phase maps divided by the total number of pixels and then normalized to 100. As the counts/pixel increase, the limit of the percent difference seems to be $\sim 0.5 \%$ instead of $0.0 \%$. The difference images calculated from spectral images of higher counts/pixel (e.g., 1262) only have pixels along phase boundaries, likely owing to subtle inconsistencies in SEM stage motion when repeating the grid acquisitions. Therefore, an SEM with perfectly repeatable stage motions would enable the acquisition of phase map montages with a $0 \%$ difference.

Fig. 1 shows examples of wt\% map montages and phase map montages acquired at varying counts/pixel. As expected from counting statistics, the images become less noisy as the counts/pixel increase. The count richness needed to meaningfully extract maps depends on the map type being extracted and what information is needed. For phase mapping, the "percent difference" metric was used to determine at what threshold adding more data has a negligible effect on improving the phase map. At 25 counts/pixel, all three phases present in this area of the sample are correctly identified. The correct identification of phases present in a sample may be achieved with very low counts when using the COMPASS algorithm. Fig. 2 (bottom) shows that the phase maps are negligibly improved after $\sim 300$ counts/pixel have been acquired, meaning that all phases have been identified in their proper locations. For low-Ca pyroxene, individual pixel compositions yield good stoichiometry $\left([\mathrm{Mg}, \mathrm{Fe}] \mathrm{SiO}_{3}\right)$ after $\sim 2,500$ counts/pixel. However, when considering the results of any quantitative elemental map, it is important to remember the role of counting statistics. An accurate and precise quantitative map, in which counting statistics are minimized to $\sim 1 \%$, requires several tens of thousands of counts per pixel as documented by [5]. For most samples, the most practical way to completely describe a very large area map is to acquire $~ 100$ counts/pixel, use COMPASS-based phase mapping to determine the identity and distribution of phases present, quantify (with standards, if possible) a spectrum summed from many pixels representing each 
phase for each phase to achieve accurate and precise analyses, and rely on quantitative elemental maps to investigate more subtle elemental distributions such as gradational zoning.

\section{References:}

[1] SM Seddio and PK Carpenter, Microsc. Microanal. 23 (2017).

[2] Keenan et al., Meth. of Multivariate Spectral Analysis. Pat. 6,675,106 B1. 06Jan.2004.

[3] Keenan et al., Apparatus and Sys. for Multivariate Spectral Analysis. Pat. 6,675,106 B1. 06Jan.2004.

[4] WS Rasband, ImageJ, US Nat. Inst. Health (1997-2016) http://imagej.nih.gov/ij/

[5] SM Seddio, Microsc. Microanal. 21 (2015) 2093.

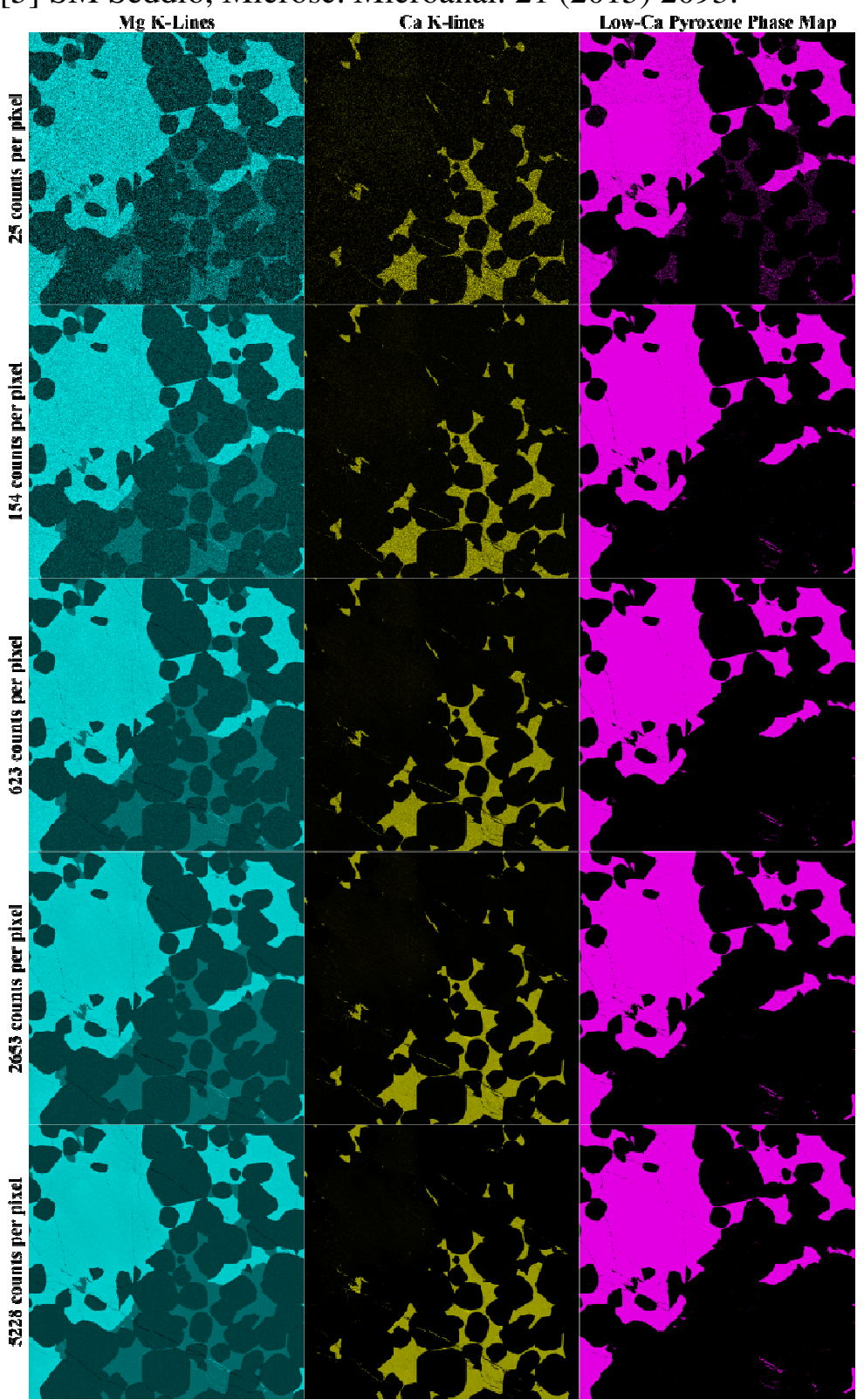

Figure 1. Montages acquired with varying counts/pixel. $\mathrm{Mg} w \mathrm{t} \%, \mathrm{Ca}$ wt $\%$, and the low-Ca pyroxene phase maps are shown as examples. "Counts per pixel" is determined by taking the total counts for each spectral image montage divided by the number of pixels in each image. Width of each montage is $2.2 \mathrm{~mm}$.

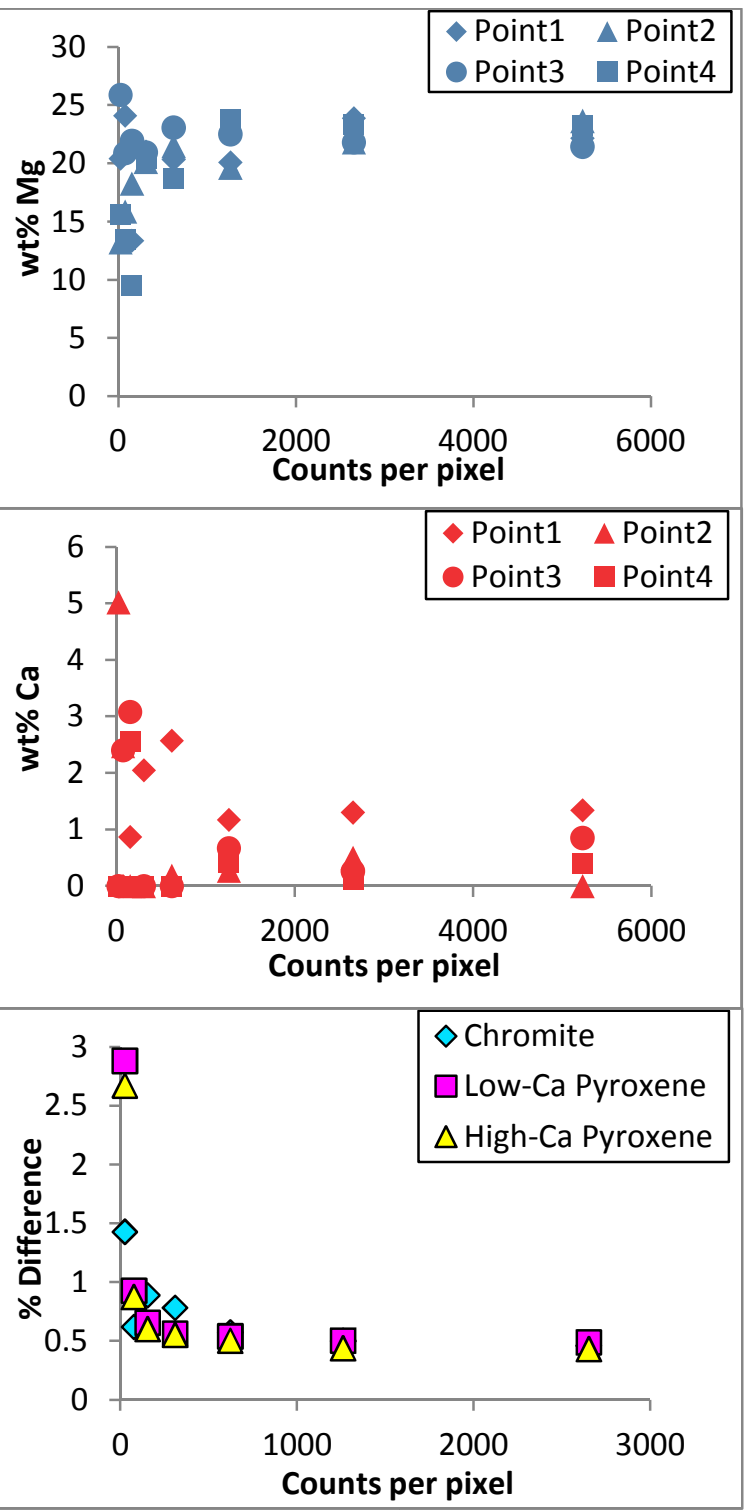

Figure 2. Top and middle: $\mathrm{Mg}$ and $\mathrm{Ca}$ concentrations calculated for the same 4 points (individual pixels) in the spectral image mosaics. All pixels represented low-Ca pyroxene. Bottom: The percent difference of phase maps acquired with different counts/pixel. 